\title{
Characterization of Particle Movement and High-resolution Separation of Microalgal Cells via Induced-charge Electroosmotic Advective Spiral Flow
}

Xiaoming Chen ${ }^{\mathrm{a}}$, Yukun Ren ${ }^{\mathrm{a}, \mathrm{b},{ }^{*} \text {, Tianyi Jiang }}{ }^{\mathrm{a}}$, Likai Hou ${ }^{\mathrm{a}}$, Hongyuan Jiang, ${ }^{\mathrm{a},}$

a. School of Mechatronics Engineering, Harbin Institute of Technology, Harbin 150001, PR China. Email: rykhit@hit.edu.cn

b. State Key Laboratory of Robotics and System, Harbin Institute of Technology, Harbin 150001, PR China. E-mail: jhy_hit@hit.edu.cn

Section 1. Schematics of whole device

Section 2. Numerical method

Section 3. Computational model

Section 4. Separation simulation

Section 5. Device fabrication

Section 6. Property of microalgal cells

Section 7. Influences of voltage and frequency on particle trajectories

Section 8. DEP properties of samples

Section 9. Frequency and voltage dependence of capturing and releasing time

Section 10. Measure method of angular velocity

Section 11. Transport of heavy and large single particle

Section 12. Fabrication of microdroplets

Section 13. Formation process of droplet system

Section 14. Flow-field distribution in the microchannel

Section 15. 3-D surface plots

Section 16: Photographs of collected Oocystis sp.

Section 17: Supplementary Movies 
Section 1. Schematics of whole device

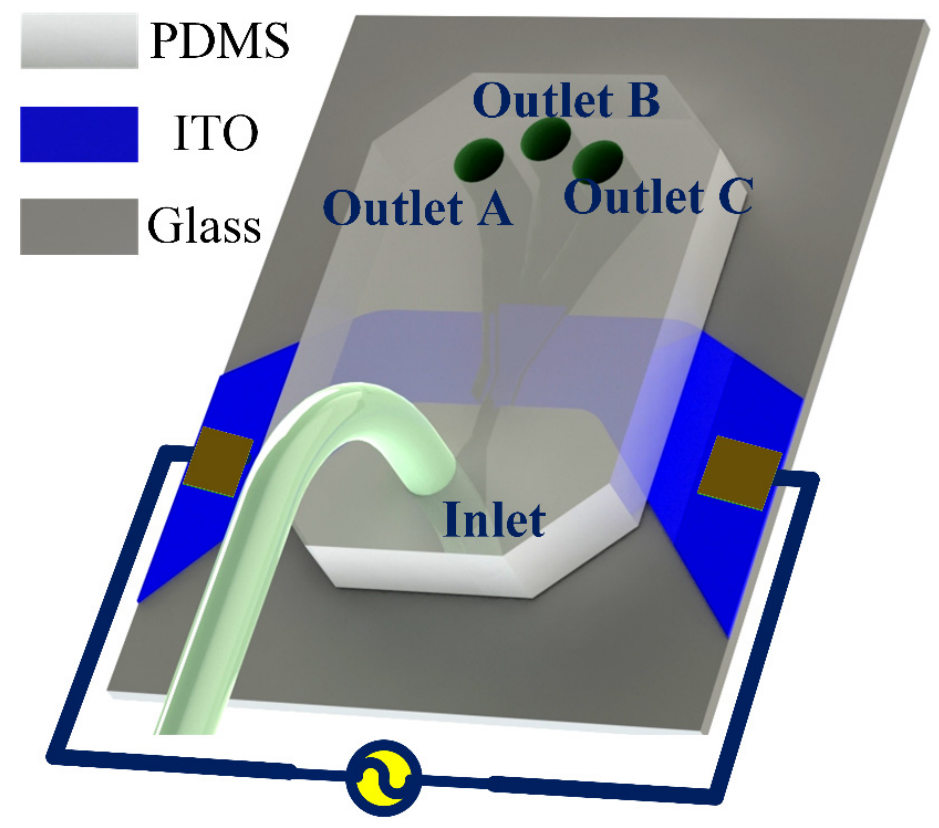

Figure S1 Schematics of device. 


\section{Section 2. Numerical method}

In the computational model, left driving electrode was applied an AC signal with a frequency $\omega=2 \pi f$ and amplitude $A_{e x}, \phi=\phi\left(A_{e x}, \omega\right)=A_{e x} \cos (\omega t+\theta)=\operatorname{Re}\left(A_{e x} e^{j \theta} e^{j \omega t}\right)=\operatorname{Re}\left(\tilde{\phi}_{e x} e^{j \omega t}\right)$, and right is grounded $\phi=0$. We considered that the fluid domain has homogeneous bulk salt concentration with the conductivity of $\sigma_{m}$. In this scenario, the distribution of electrostatic potential $V=\phi(A, \omega)$ in the fluid domain can be calculated by Laplace equation: ${ }^{1}$

$$
\nabla^{2} \phi=0
$$

Under the influence of electric field, equal positive and negative ions are driven toward different sides of electrode like charging process of a skin capacitance, forming induced double layer (IDL). Normal current continuity can elucidate conservation of diffuse charge in the IDL and describe this interfacial jump condition. $^{2}$

$$
\sigma_{m} \cdot \boldsymbol{n} \nabla \phi=C_{0} \frac{\partial\left(\phi_{b}-\phi_{0}\right)}{\partial t}
$$

where, $\phi_{b}(A, \omega)$ and $\phi_{0}(A, \omega)$ are the potential in the fluid domain and at the gate electrode, $C_{0}$ indicates the interfacial total capacitance, $\boldsymbol{n}$ represents unit normal vector. Meanwhile, the channel walls were employed following boundary conditions: ${ }^{3}$

$$
\boldsymbol{n} \cdot \nabla \phi=0
$$

The zeta potential on their gate electrode can be calculated by: ${ }^{2}$

$$
\zeta=\left(\phi_{0}-\phi_{b}\right) /(1+\delta)
$$

where, $\delta$ stands for the surface capacitance ratio of diffuse layer and compact stern layer.

The electric-field component parallel to the gate electrode drives excessive counterions within the diffuse layer to move along or against the electric field. ${ }^{3}$

$$
\boldsymbol{E}_{\boldsymbol{t}}=\boldsymbol{E}-\boldsymbol{E} \cdot \boldsymbol{n} \cdot \boldsymbol{n}=\nabla \phi-\nabla \phi \cdot \boldsymbol{n} \cdot \boldsymbol{n}
$$

When $1 / \omega$ is longer than the charging time of IDL, electroosmotic flow (EOF) occurred on the gate electrode can be described by the Helmholtz-Smoluchowski relation: ${ }^{2}$

$$
\begin{aligned}
\left\langle\boldsymbol{u}_{E O F}\right\rangle & =-\frac{\varepsilon_{m}\left\langle\zeta \boldsymbol{E}_{\boldsymbol{t}}\right\rangle}{\eta}=-\frac{\varepsilon_{m}\left\langle\tilde{\zeta} \tilde{\boldsymbol{E}}_{t}^{*}\right\rangle}{\eta} \\
& =\frac{\varepsilon_{m}}{2 \eta(1+\delta)} \operatorname{Re}\left[\left(\tilde{\phi}_{b}-\tilde{\phi}_{0}\right)(\nabla \tilde{\phi}-\nabla \tilde{\phi} \cdot \boldsymbol{n} \cdot \boldsymbol{n})^{*}\right]
\end{aligned}
$$

where, $\varepsilon_{m}$ and $\eta$ represent the permittivity and dynamic viscosity of fluid domain.

Equation (S6) as the boundary condition is employed on the gate electrode. Besides, left and right electrodes are employed the slip velocity:

$$
\begin{gathered}
\left\langle\boldsymbol{u}_{L}\right\rangle=\frac{\varepsilon_{m}}{2 \eta(1+\delta)} \operatorname{Re}\left[\left(\tilde{\phi}-A_{e x}\right)(\nabla \tilde{\phi}-\nabla \tilde{\phi} \cdot \boldsymbol{n} \cdot \boldsymbol{n})^{*}\right] \\
\left\langle\boldsymbol{u}_{R}\right\rangle=\frac{\varepsilon_{m}}{2 \eta(1+\delta)} \operatorname{Re}\left[\tilde{\phi}(\nabla \tilde{\phi}-\nabla \tilde{\phi} \cdot \boldsymbol{n} \cdot \boldsymbol{n})^{*}\right]
\end{gathered}
$$

Besides, the rest channel walls are defined as no slip walls $\boldsymbol{u}=0$. Moreover, the fluid domain is governed by full Stokes equation. 


$$
\begin{gathered}
\nabla \boldsymbol{u}_{E O F}=0 \\
-\nabla p+\nabla\left(\eta\left(\nabla \boldsymbol{u}_{E O F}+\left(\nabla \boldsymbol{u}_{E O F}\right)^{T}\right)\right)=\rho \frac{\partial \boldsymbol{u}_{E O F}}{\partial t}
\end{gathered}
$$

In this scenario, we can obtain the distribution of fluid velocity caused by induced charge electroosmotic (ICEO) flow $\boldsymbol{u}_{E O F}(A, \omega)$.

When particles are rotated by ICEO vortices at the edges of gate electrode with electric field gradient, particle trajectories will be reshaped duo to the Maxwell-Wagner interfacial polarization. Dielectrophoresis (DEP) force for a sphere particle with a diameter $d$ can be calculated by: ${ }^{4}$

$$
\left\langle\boldsymbol{F}_{D E P}\right\rangle=\pi \varepsilon_{m} d^{3} \operatorname{Re}[K(\omega)] \nabla|\boldsymbol{E}|^{2} / 8
$$

where, $d$ indicates the diameter of microparticles, $\operatorname{Re}[K(\omega)]$ represents the real part of the complex Clausius Mossotti (CM) factor, which illustrates the relative polarization of particles versus that of buffer solution calculated by ${ }^{4}$

$$
\operatorname{Re}[K(\omega)]=\frac{\varepsilon_{p}^{*}(\omega)-\varepsilon_{m}^{*}(\omega)}{\varepsilon_{p}^{*}(\omega)+2 \varepsilon_{m}^{*}(\omega)}
$$

where, $\varepsilon_{p}^{*}(\omega)=\varepsilon_{p}-j \sigma_{p} / \omega$ and $\varepsilon_{m}^{*}(\omega)=\varepsilon_{m}-j \sigma_{m} / \omega$ indicate the com-plex permittivity of particle and buffer solution. $\varepsilon_{p} / \varepsilon_{m}$ and $\sigma_{p} / \sigma_{m}$ represent the real permittivity and conductivity of the particles/ medium.

Therefore, particles rotate in ICEO vortices with the velocity:

$$
\begin{aligned}
\boldsymbol{u}_{p}(A, \omega) & =\boldsymbol{u}_{E O F}(A, \omega)+\boldsymbol{u}_{\text {buoyancy }}+\boldsymbol{u}_{D E P} \\
& =\boldsymbol{u}_{E O F}(A, \omega)-\frac{d^{2}}{18 \eta}\left(\rho_{p}-\rho_{f}\right) \boldsymbol{g}+\frac{\varepsilon_{m} d^{2}}{24 \eta}\left[\operatorname{Re}[K(\omega)] \nabla|\boldsymbol{E}|^{2}\right]
\end{aligned}
$$

In this equation, the first term is the velocity caused by the fluid motion, which can be regulated by adjusting frequency and amplitude of external voltage. Motion of fluid induced fluidic drags exerting on particles in $\mathrm{Y}$ and $\mathrm{Z}$ directions, $\boldsymbol{F}_{\mathrm{Y} \text {, Drag }}$ and $\boldsymbol{F}_{\mathrm{Z}}$, Drag. The second term is velocity induced by buoyancy force $\boldsymbol{F}_{\text {Buo }}$ determined mainly by particle size and density. The third term is the velocity actuated by DEP force (negative DEP (nDEP) force, $\boldsymbol{F}_{\mathrm{nDEP}}$, and positive DEP (pDEP) force, $\boldsymbol{F}_{\mathrm{pDEP}}$ ) determined by relative polarization of particles and buffer solution, which can be tuned by controlled the frequency, amplitude of voltage, and conductivity of surrounding fluid. We can regulate the external voltage to make the particles reach diverse stable moving states and switch the moving states flexibly. 


\section{Section 3. Computational model}
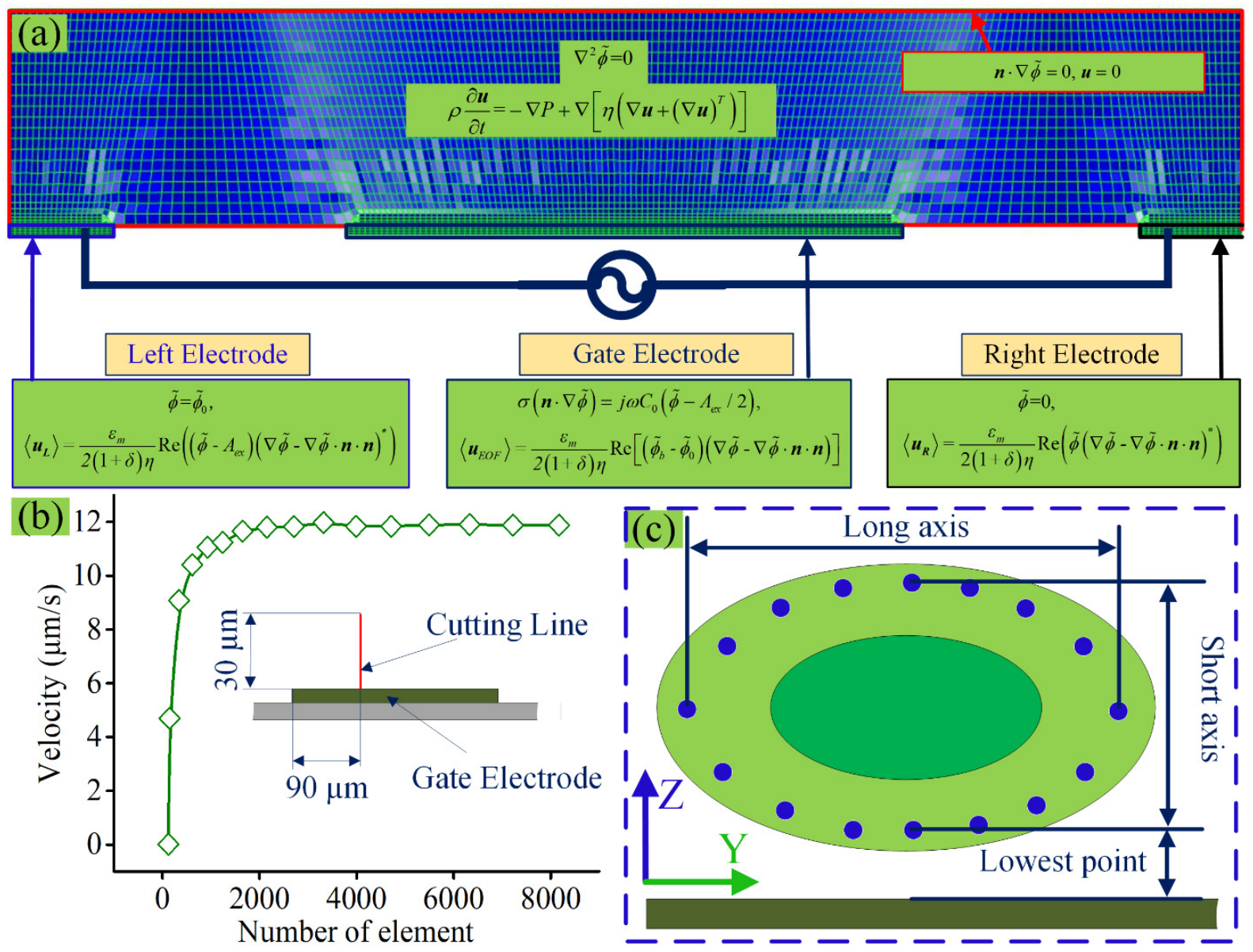

Figure S2 Computational model and index definition. (a) Computational model and employed conditions. (b) Analysis of element sensitivity. (c) Index definitions depicting the particle trajectories.

To investigate the behaviors of particles in ICEO vortices, we established the computational model, as given in Figure S2a, comprising two driving electrodes, gate electrode and fluid domain. External electric field was applied on the left and right driving electrodes. Excitement of AC electric field gave rise to creation of counter-rotating ICEO streaming on the gate electrode. Small particles were released from the fluid domain and interacted with the ICEO vortices. Under fixed work parameters, particles presented their unique and stable moving states, being captured in vortex cores, orbiting around vortex cores, or escaping to exterior of vortices, which was determined by their intrinsic properties. Remarkably, the patterns of ICEO streaming can be tuned by modulating the working parameters, accomplishing precise adjustment and flexible switch of particle states. In Figure S2a, computational model consists of $600 \mu \mathrm{m}$ wide and $105 \mu \mathrm{m}$ high fluid domain. Two $50 \mu \mathrm{m}$ wide driving electrodes and a $270 \mu \mathrm{m}$ wide gate electrode are designed on the floor of fluid domain, spaced $115 \mu \mathrm{m}$ apart. Moreover, the governing equations and boundary conditions employed in the computational model are also exhibited in Figure S2a. We utilized the mapping method to mesh the computational model and refined the elements close to the fluid/electrode interfaces. Analysis of element sensitivity was conducted by evaluating the maximum Z-direction velocity along the cutting line above gate electrode (defined in Figure S2b). From Figure S2b, at the number of 
elements exceeding $3.988 \times 10^{3}$, the maximum vertical velocity reaches stable at $11.879 \mu \mathrm{m} / \mathrm{s}$. To save calculation time, we determined the simulation model with divided element of $3.988 \times 10^{3}$. In addition, proposed computational model coupling electric and flow flied was applied to provide quantitative illustrations of particle trajectories driven by ICEO streaming, including long axis, short axis, and the lowest point, which are defined in Figure S2c. 


\section{Section 4. Separation simulation}
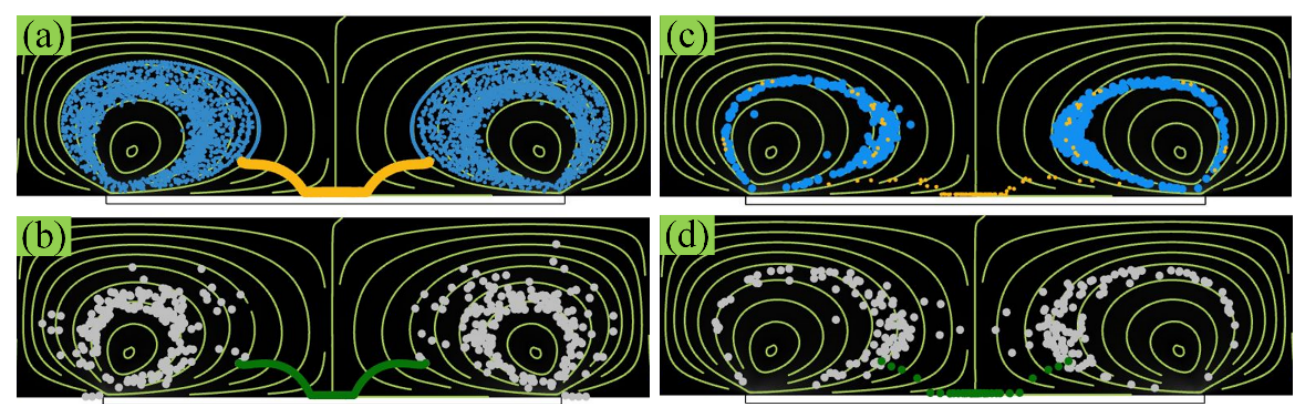

Figure S3 Simulation of particle separation based on different characteristics. (a) Size-based separation at $\operatorname{Re}[K(\omega)]>0$. (b) Density-based separation at $\operatorname{Re}[K(\omega)]>0$. (c) Size-based separation at $\operatorname{Re}[K(\omega)]<0$. (d) Density-based separation at $\operatorname{Re}[K(\omega)]<0$.

We conducted the numerical simulations to investigate the performance of our method in size- and density-based separation of particles with different DEP properties.

In the first place, we investigated the size-based separation at $\operatorname{Re}[K(\omega)]=-4.63$ (Figure S3a). In the simulation, 4- $\mu \mathrm{m}$ blue and $8-\mu \mathrm{m}$ yellow particles shared the same density $\rho=1100 \mathrm{~kg} / \mathrm{m}^{3}$. In the ICEO vortices, $4-\mu \mathrm{m}$ blue particles were readily to be elevated by ICEO vortices, whereas 8 - $\mu \mathrm{m}$ yellow particles couldn't be captured by ICEO vortices and were concentrated in the center of gate electrode at $f=300 \mathrm{~Hz}$ and $A=4 \mathrm{~V}$.

In the second place, we explored the performance of our method in the density-based separation at $\operatorname{Re}[K(\omega)]=-4.63$ (Figure S3b). In the simulation, white and yellow particles shared the same size, $d=6 \mu \mathrm{m}$. In the ICEO vortices, white particles were readily elevated and then captured by ICEO vortices due to low density $\rho=1050 \mathrm{~kg} / \mathrm{m}^{3}$ at $f=300 \mathrm{~Hz}$ and $A=5.2 \mathrm{~V}$. In contrast, green particles can't be captured by ICEO vortices and aligned in the middle of ICEO vortices for their high density $\rho=1400 \mathrm{~kg} / \mathrm{m}^{3}$.

In the third place, we validated the feasibility of our method in the size-based separation at $\operatorname{Re}[K(\omega)]=0.4$ (Figure $\mathrm{S} 3 \mathrm{c}$ ). In the simulation, 12- $\mu \mathrm{m}$ blue particles were attracted to gate electrode edge under strong pDEP force and rotated with ICEO vortices. At the same time, weak pDEP force couldn't overcome the fluidic drag force, and 6- $\mu \mathrm{m}$ yellow particles were transported to center of gate electrode by the ICEO flow at $f=300 \mathrm{~Hz}$ and $A=5.6 \mathrm{~V}$. Blue and yellow particles shared the same density $\rho=1100 \mathrm{~kg} / \mathrm{m}^{3}$.

In the fourth place, we proved the effectiveness of our approach in the density-based separation at $\operatorname{Re}[K(\omega)]=0.4$ (Figure S3d). In the simulation, white and yellow particles shared the same size, $d=8 \mu \mathrm{m}$. At $f=300 \mathrm{~Hz}$ and $A=6 \mathrm{~V}$, white particles were easy to be elevated by ICEO vortices and then moved with ICEO vortices for low density $\rho=1200 \mathrm{~kg} / \mathrm{m}^{3}$. Under the same condition, green particles can't be captured by ICEO vortices and were aligned in the middle of ICEO vortices for high density $\rho=1500 \mathrm{~kg} / \mathrm{m}^{3}$. 


\section{Section 5. Device fabrication}

Fabrication of our device mainly includes three steps: PDMS channel fabrication, ITO electrode pattern, and alignment and bonding. In the process of PDMS-based channel fabrication, we needed to prepare a clean glass substrate with the width of $5 \mathrm{~cm}$ and length of $6 \mathrm{~cm}$ (Figure S4a). After 1-mL DI water covering the glass substrate, a layer of dry film (Riston SD238, Dupont, USA) with the thickness of $35 \mu \mathrm{m}$ was pasted on the substrate. Afterward, glass substrate with dry film was proceed in the laminator under the hot mode for four times (Figure S4b). Then, another layer of dry film was pasted on the first dry film by repeating last step, and the second layer of dry film was compressed using the laminator under the cold mode for four times (Figure S4c). After this, dry films were covered by the photo mask, and then exposed to UV light for $12 \mathrm{~s}$ (Figure $\mathrm{S} 4 \mathrm{~d}$ ). In the $1.5 \% \mathrm{Na}_{2} \mathrm{CO}_{3}$ solution, dry films were developed into designed structure (Figure $\mathrm{S} 4 \mathrm{e}$ ). In the vacuum tanks, the dry-film mold was processed to reduce its surface energy with 1H, 1H, 2H, 2H-perfluorooctyltrichlorosilane (Sigma Aldrich, USA) (Figure S4f). 10:1 mixture of a Sylgard 184 Silicone Elastomer Base and a Sylgard 184 Silicone Elastomer Curing Agent (Dow Corning, Inc, MI) were poured on the dry-film mold (Figure S4g). Because there were many air bubbles in the PDMS, we evacuated the air bubble in the vacuum tanks (Figure S4h). In the next step, we needed to bake the PDMS in a preheated oven at $80{ }^{\circ} \mathrm{C}$ for 1 hour (Figure S4i). Finally, we peeled the PDMS channel from the dry-film mold (Figure $\mathrm{S} 4 \mathrm{j}$ ).

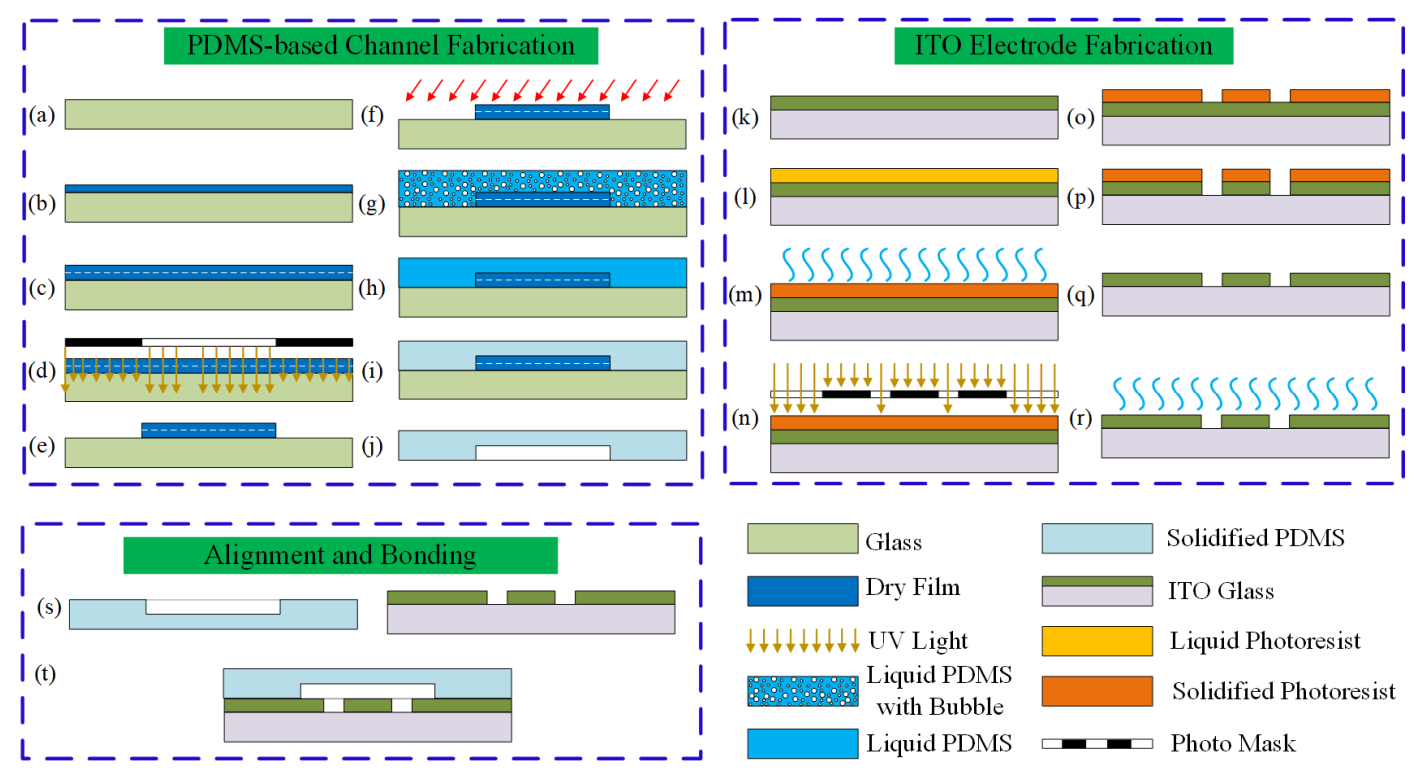

Figure S4 Detail of device fabrication.

In the ITO electrode fabrication, we firstly prepared a clean glass with ITO film (Figure S4k). Positive photoresist (AZ4620) was coated on the ITO film at the speed of $3000 \mathrm{rad} / \mathrm{min}$ for $45 \mathrm{~s}$ (Figure S4l). On the hot plate, ITO glass was baked at $100^{\circ} \mathrm{C}$ for 6 mins to solidify coated positive photoresist (Figure $\mathrm{S} 4 \mathrm{~m})$. We covered the positive photoresist with the photo mask and exposed them to the UV light for 180 $\mathrm{s}$ (Figure S4n). In the developer (ZX-238), positive photoresist was fabricated to desired structures (Figure S4o). Afterward, we submerged ITO glass into etching solution to process the ITO film to desired structure (Figure S4p). We removed positive photoresist in the acetone solution for 3 mins (Figure S4q). Finally, we cleaned the ITO electrode and conducted the drying process in the oven at $50{ }^{\circ} \mathrm{C}$ for 1 hour (Figure 
S4r).

Prior to alignment and bonding, we conducted a surface treatment of oxygen plasma for the PDMS channel and ITO electrode (Figure S4s). Under the microscope, we aligned the PDMS channel and ITO electrode in 5 mins. Aligned device were bonded at the room temperature for 2 hours (Figure S4t). 


\section{Section 6. Property of microalgal cells}

\section{DEP performance of microalgal cells}
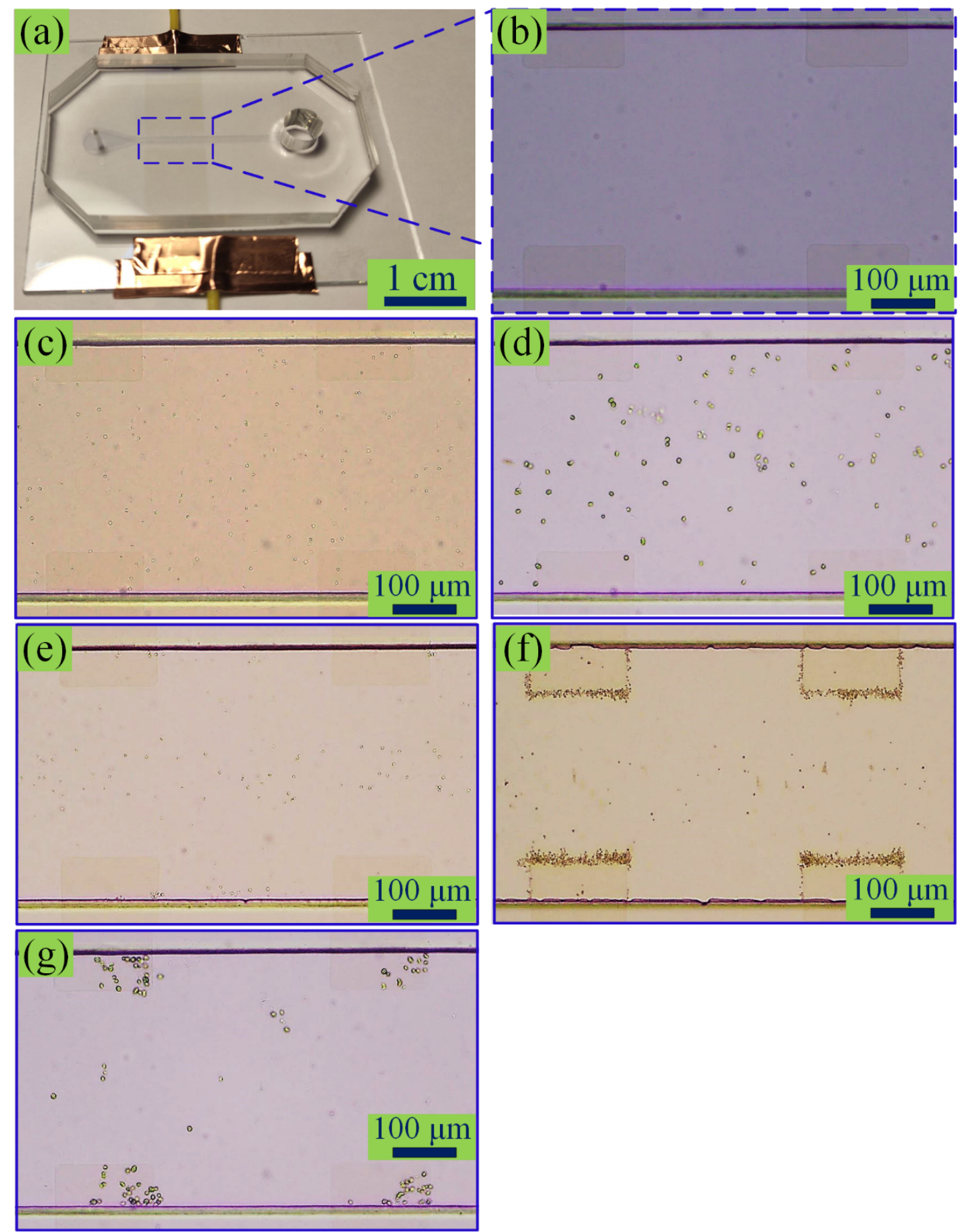

Figure S5 DEP performances of microalgal cells in the $\mathrm{KCl}$ solution with the conductivity of $10 \mu \mathrm{S} / \mathrm{cm}$. (a) Photograph of device. (b) Configuration of microchannel. (c) and (d) States of Chlorella vulgaris and Oocystis sp. were distributed randomly in the microchannel without electric field. (e) Chlorella vulgaris was pushed away from electrodes by nDEP force at $f=300 \mathrm{~Hz}, A=7.5 \mathrm{~V}$. (f) Chlorella vulgaris was attracted toward the electrodes under the pDEP force at $f=100 \mathrm{kHz}, A=75 \mathrm{~V}$. (g) Oocystis sp. was attracted toward electrodes due to pDEP force at $f=300 \mathrm{~Hz}$ and $A=7.5 \mathrm{~V}$. 
Actual photograph of the device is given in Figure S5a, and microchannel configuration is given in Figure S5b. Chlorella vulgaris and Oocystis sp. were distributed in the microchannel randomly with no electric field as shown in Figure S5c and d. At $f=300 \mathrm{~Hz}$ and $A=7.5 \mathrm{~V}$, Chlorella vulgaris experienced $\mathrm{nDEP}$ force and they were repelled away from electrodes (Figure S5e). As a result, they were focused in the center of microchannel. By modulating frequency to $f=5.5 \mathrm{kHz}$, Chlorella vulgaris began to undergo pDEP force, and they were attracted to the electrodes. At $f=100 \mathrm{kHz}$ and $A=75 \mathrm{~V}$, all the Chlorella vulgaris moved toward and then were adhered to the electrodes (Figure S5f). At $f=100 \mathrm{~Hz}$ to $100 \mathrm{kHz}$, Chlorella vulgaris always experienced pDEP force. Experimental snapshot illustrating the performance of Oocystis sp. at $f=300 \mathrm{~Hz}$ and $A=7.5 \mathrm{~V}$ is given in Figure $\mathrm{S} 5 \mathrm{~g}$.

Size of microalgal cells

Table S1 Sizes of different-cell Oocystis sp.

\begin{tabular}{|c|c|c|c|c|c|}
\hline Cell Number & 1 & 2 & 3 & 4 & 5 \\
\hline Size $(\mu \mathrm{m})$ & $8.02 \sim 9.51$ & $11.30 \sim 12.8$ & $13.64 \sim 16.67$ & $18.29 \sim 21.05$ & $21.77 \sim 24.95$ \\
\hline Cell Number & 6 & 7 & 8 & 10 & 12 \\
\hline Size $(\mu \mathrm{m})$ & $24.44 \sim 27.23$ & $29.92 \sim 31.07$ & $33.28 \sim 35.68$ & $55.54 \sim 57.24$ & $58.26 \sim 60.13$ \\
\hline
\end{tabular}




\section{Section 7. Influences of voltage and frequency on particle trajectories}

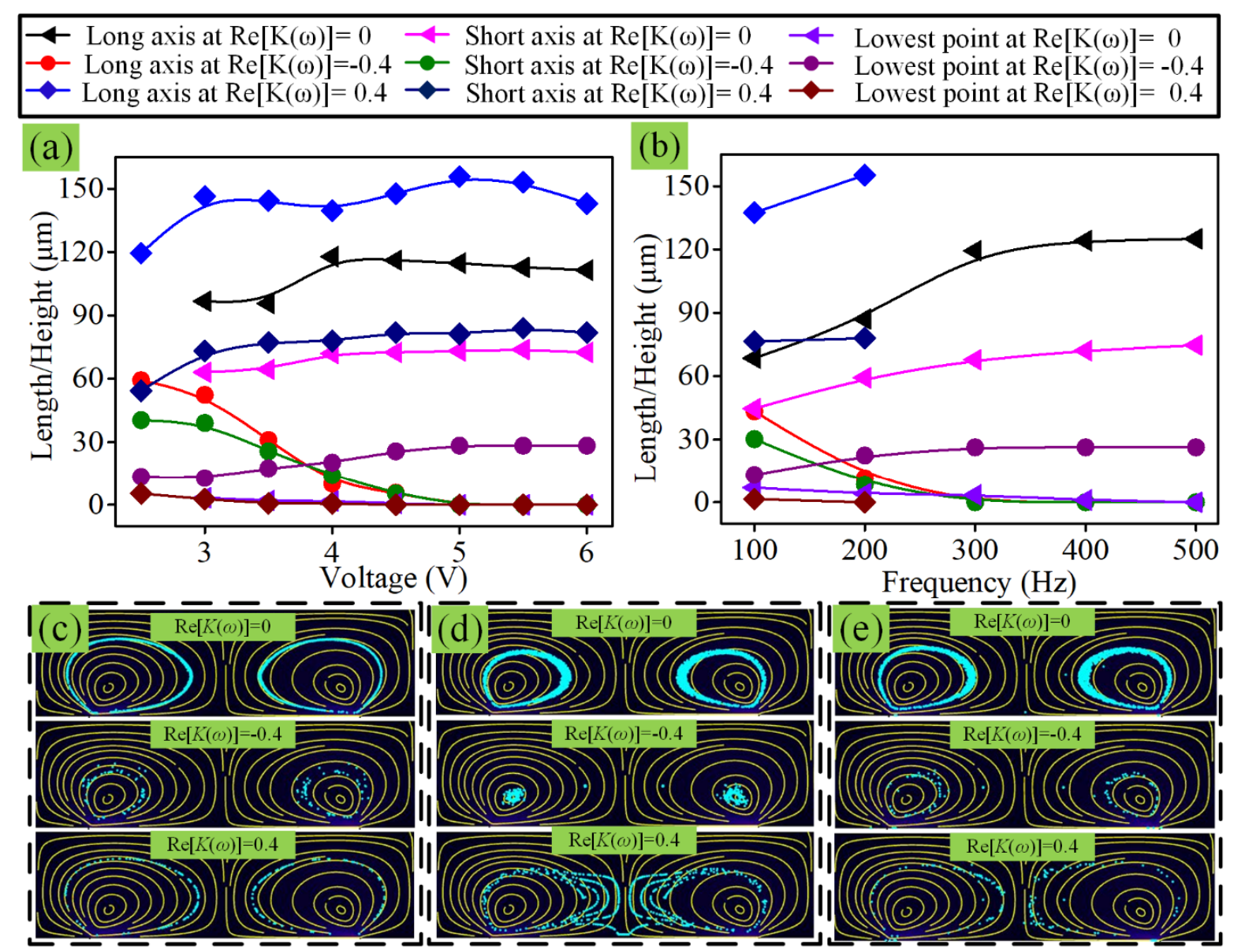

Figure S6 Voltage and frequency effect on particle trajectories. (a) Voltage and (b) frequency influence on particle orbits.

We investigated the voltage-intensity dependences numerically (Figure S6a) at $f=200 \mathrm{~Hz}, d=5 \mu \mathrm{m}$, and $\rho=1050 \mathrm{~kg} / \mathrm{m}^{3}$. By increasing the voltage from 2.5 to $6 \mathrm{~V}$, the ranges of particle motion, at $\operatorname{Re}[K(\omega)]=0$ and 0.4, were gradually enlarged. The long axis and short axis were increased from about 96.67 to $117.79 \mu \mathrm{m}$ and from 63.09 to $73.82 \mu \mathrm{m}$ at $\operatorname{Re}[K(\omega)]=0$. At $\operatorname{Re}[K(\omega)]=-0.4$, the long axis and short axis both presented downward trend from roughly 59.32 and 40.45 to $0 \mu \mathrm{m}$. Under above two conditions, particle moving regions were consistently magnified to the gate electrode. Conversely, at $\operatorname{Re}[K(\omega)]=0.4$, the long axis and short axis both experienced a marked increase from about 119.46 to $155.7 \mu \mathrm{m}$ and from 54.36 to $83.89 \mu \mathrm{m}$, concentrating the particle only in vortex cores. Simulated particle tracings at $A=3$ and $5 \mathrm{~V}$ are exhibited in Figure S6c and d.

Frequency effect on the particle motion was also performed at $A=4 \mathrm{~V}, d=5 \mu \mathrm{m}$ and $\rho=1100 \mathrm{~kg} / \mathrm{m}^{3}$ (Figure $\mathrm{S} 6 \mathrm{~b})$. At $f=100 \sim 600 \mathrm{~Hz}$, the long axis and short axis of particles $(\operatorname{Re}[K(\omega)]=0)$ were steadily rise from 68.55 to $125.03 \mu \mathrm{m}$ and from 44.6 to $74.71 \mu \mathrm{m}$, and orbit gradually contacted the gate electrode. At $f>200 \mathrm{~Hz}$, particles $(\operatorname{Re}[K(\omega)]=0.4)$ began to escape from the vortices to the gate-electrode centerline duo to the enlarged orbit. Due to $\boldsymbol{F}_{\mathrm{nDEP}}(\operatorname{Re}[K(\omega)]=-0.4)$, particle orbit witnessed a consistent drop (long axis: from 43.35 to $0 \mu \mathrm{m}$, short axis: from 30.19 to $0 \mu \mathrm{m}$ ) at $f=100 \sim 500 \mathrm{~Hz}$. Simulated particle tracing at $f=200$ $\mathrm{Hz}$ are exhibited in Figure S6e. 


\section{Section 8. DEP properties of samples}
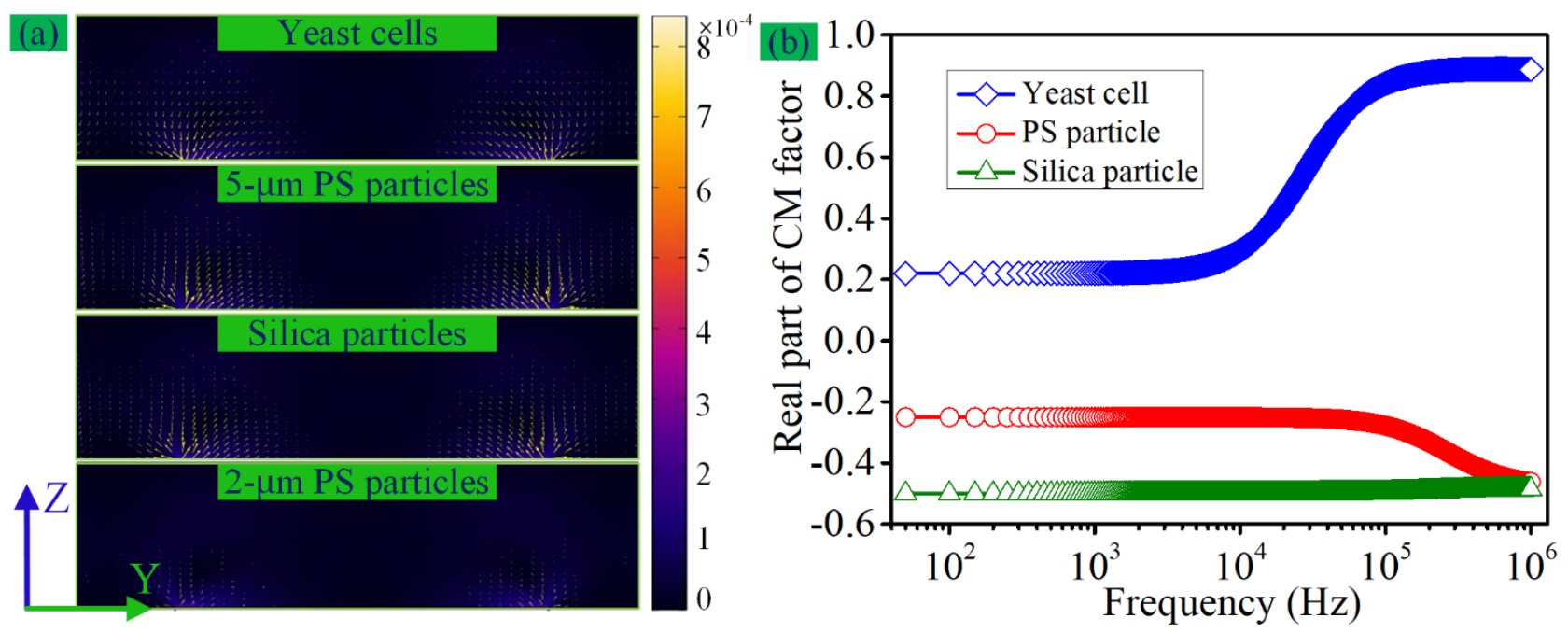

Figure S7 DEP properties of yeast cells, 5- $\mu \mathrm{m}$ PS, silica, and 2- $\mu \mathrm{m}$ PS particles. (a) DEP-induced velocity of said samples in the ICEO vortices. (b) Real part of CM factor of said particles versus frequency at the conductivity of $10 \mu \mathrm{S} / \mathrm{cm}$.

We studied the DEP property of yeast cells, 5- $\mu \mathrm{m}$ PS, silica, and 2- $\mu \mathrm{m}$ PS particles. Velocities induced by DEP forces of said small particles are given in Figure S7a at $f=200 \mathrm{~Hz}, \sigma=10 \mu \mathrm{S} / \mathrm{cm}$ and $A=3 \mathrm{~V}$. According to Figure S7a, nDEP force provides an upward repulsive force, reducing moving range of yeast cells, whereas pDEP force offers a downward attractive force, enlarging orbits of colloid particles. Real parts of CM factors of silica, PS particles and yeast cells against frequency at $\sigma=10 \mu \mathrm{S} / \mathrm{cm}$ are shown in Figure S7b. 


\section{Section 9. Frequency and voltage dependence of capturing and releasing time}

Firstly, we investigated the frequency effect on capturing time. At $f=200 \mathrm{~Hz}$ and $A=7.5 \mathrm{~V}, 2-\mu \mathrm{m}$ PS and $4-\mu \mathrm{m}$ silica particles presented good separation state. At $A=15 \mathrm{~V}$, we studied frequency dependence of capturing time, which is given in Figure S8a. With increasing the frequency from 30 to $190 \mathrm{~Hz}$, the capturing time saw a consistent drop from 19 to $6.07 \mathrm{~s}$. One reasonable explanation for this trend is that attractive force between particles and gate electrode was reduced with increasing the frequency. When the frequency is over $200 \mathrm{~Hz}$, capturing time present increase trend. At $f=100 \mathrm{~Hz}$, we studied the voltage influence on capturing time (Figure S8b). With increasing the voltage from 13.75 to $20 \mathrm{~V}$, the capturing time exhibited a downward trend from $7.12 \mathrm{~s}$ to $3.14 \mathrm{~s}$. Because the fluid velocity induced by ICEO will be enhanced with increasing the voltage.

Secondly, we explored the frequency and voltage effects on the releasing times. We modulated the parameters to $f=100 \mathrm{~Hz}, A=15 \mathrm{~V}$ to capture silica particles using 2- $\mu \mathrm{m}$-PS-particle-fluid ICEO vortices, and then we tuned the voltage to $7.5 \mathrm{~V}$ to investigate the frequency effect on the releasing time (Figure S8c). In the range of $f=30$ to $190 \mathrm{~Hz}$, releasing time experienced a drop from 27.6 to $14.5 \mathrm{~s}$. By increasing the frequency to $210 \mathrm{~Hz}$, releasing time increased gradually from 14.7 to $15.5 \mathrm{~s}$. Voltage dependence of releasing time at $f=100 \mathrm{~Hz}$ is given in Figure S8d. With increasing the voltage from 5 to $12.5 \mathrm{~V}$, releasing time presented a downward trend from 33.1 to $5.9 \mathrm{~s}$.
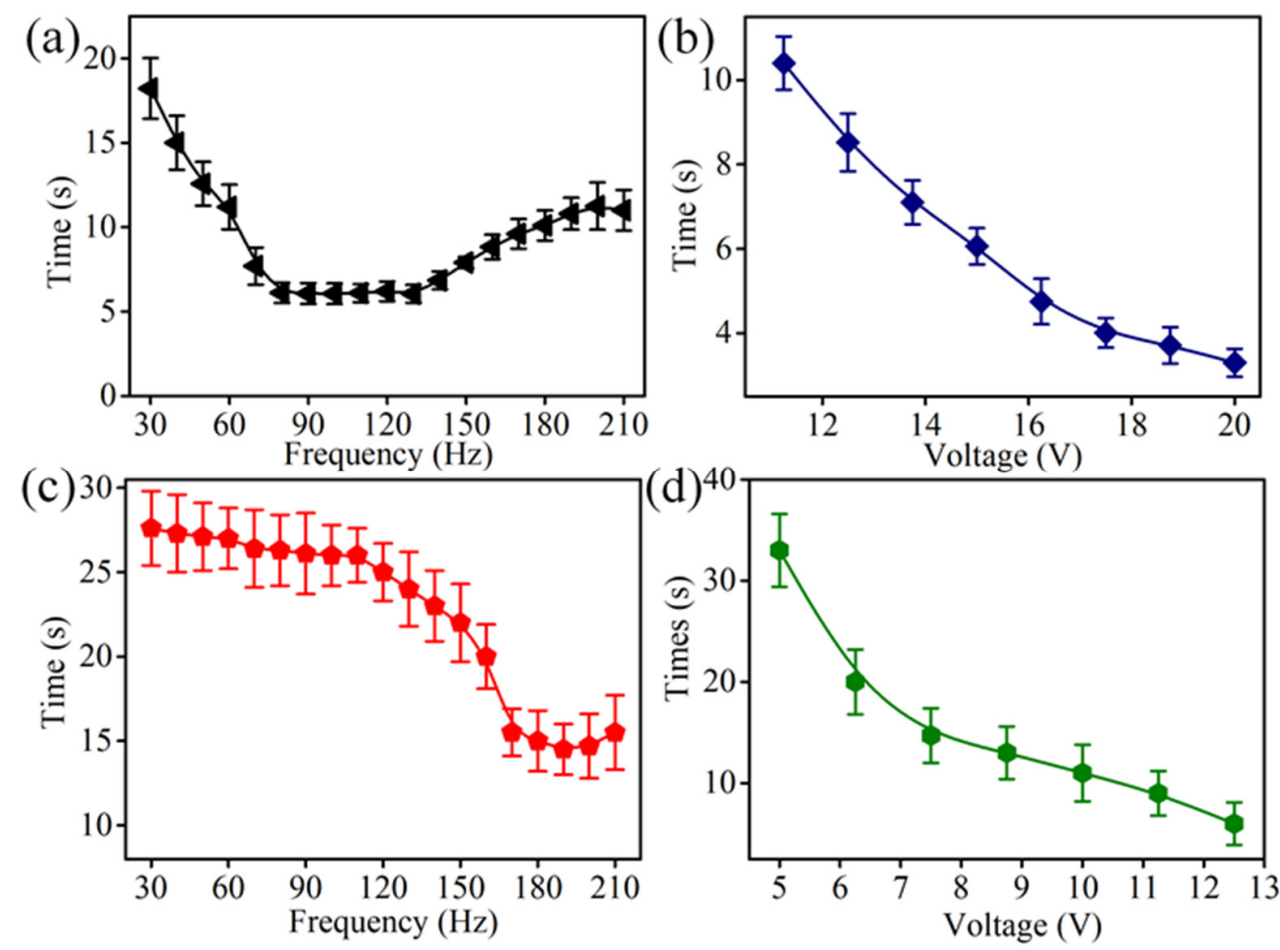

Figure S8 Frequency and voltage dependences of response time. (a) and (b) Frequency and voltage effects on capturing time. (c) and (d) Frequency and voltage influences on releasing time. 


\section{Section 10. Measure method of angular velocity}

In the experiment, we recorded the motion of silica particles in different particle-fluid phases. Then, we obtained the total time, $t_{\mathrm{i}}$, that silica particles rotated around vortex cores for 20 cycles by watching the video in the software of Adobe Premiere Pro CC 2017. This procedure was repeated for 3 times, and we obtained $t_{1}, t_{2}$, and $t_{3}$. Then, we calculated the angular velocity of silica particles according to following equation.

$$
\omega=\frac{1}{3}\left(\frac{2 \pi \times 20}{t_{1}}+\frac{2 \pi \times 20}{t_{2}}+\frac{2 \pi \times 20}{t_{3}}\right)
$$




\section{Section 11. Transport of heavy and large single particle}

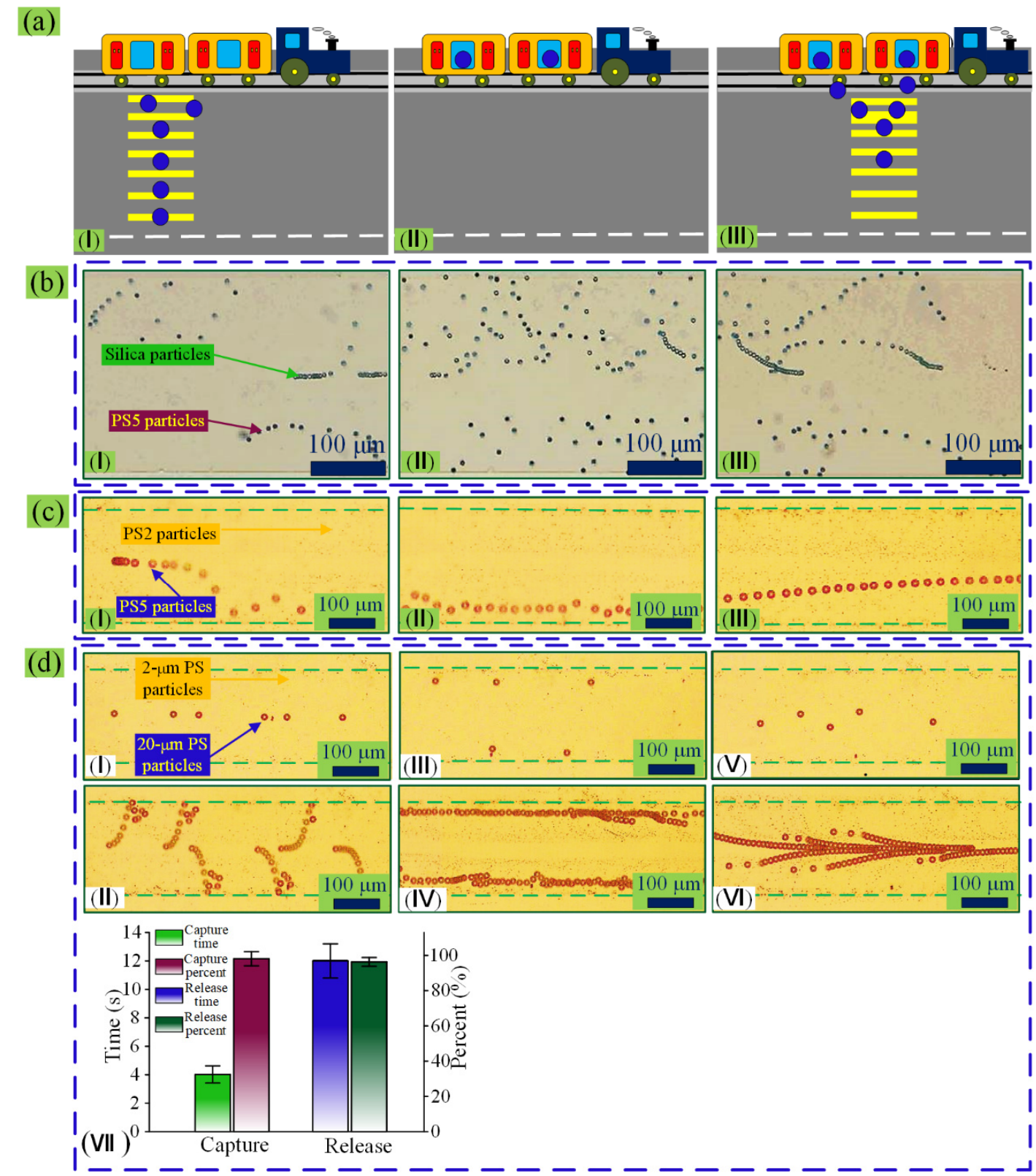

Figure S9 Transport of heavy and large particles via particle-enhanced ICEO vortices. (a) Schematic diagrams demonstrating transport process of cumbersome cargoes. (b) Procedure and exemplary micrographs of silica particle transport. (c) Collective motion of $20-\mu \mathrm{m}$ PS particles. (d) Transport process of $20-\mu \mathrm{m}$ PS particles.

We explored the performance of particle-fluid ICEO (PFICEO) vortices in the transport of heavy and large particles. Schematic diagrams elucidating the workflow are given in Figure S9a. At starting point, passengers take the train (Figure S9aI). Train carries the passengers to the destination in the trip (Figure S9aII). At finish point, passengers get off the train (Figure S9aIII). Then, we utilized the 5- $\mu$ mPS-particle-enhanced ICEO vortices to validate effectiveness of this proposition. Silica particles are loaded by particle-fluid ICEO (PFICEO) advective spiral flow at start point by regulating voltage from $f=150 \mathrm{~Hz}$ and $A=6 \mathrm{~V}$ to $f=50 \mathrm{~Hz}$ and $A=6.5 \mathrm{~V}$ (Figure S9bI). Next, augmented advective spiral flow carried silica particles to finish point (Figure S9bII). At designed position, silica particles left the 
reinforced convective flow for their destinations by tuning the voltage to $f=150 \mathrm{~Hz}$ and $A=6 \mathrm{~V}$ (Figure S9bIII). Meanwhile, 5- $\mu \mathrm{m}$ PS particles obeyed prescribed trajectories without any effect on the motion of silica particles. To further test the capability of PFICEO vortices, we used the 2- $\mu \mathrm{m}$-PS-particleenhanced ICEO vortices to transport large-sized particles, 20- $\mu \mathrm{m}$ PS particles (Video S2). Transport process of $20-\mu \mathrm{m}$ PS particles is given in Figure S9c. Collective motion of $20-\mu \mathrm{m}$ PS particles is shown in Figure S9dI-VI. Due to large size, 20- $\mu \mathrm{m}$ PS particles was difficult to be captured by ICEO vortices, but small $2-\mu \mathrm{m}$ PS particles was readily trapped by vortices in the separation process at $f=150 \mathrm{~Hz}$ and $A=6 \mathrm{~V}$. Capture and release times/ efficiency of PFICEO vortices are given in Figure S9dVII. 


\section{Section 12. Fabrication of microdroplets}
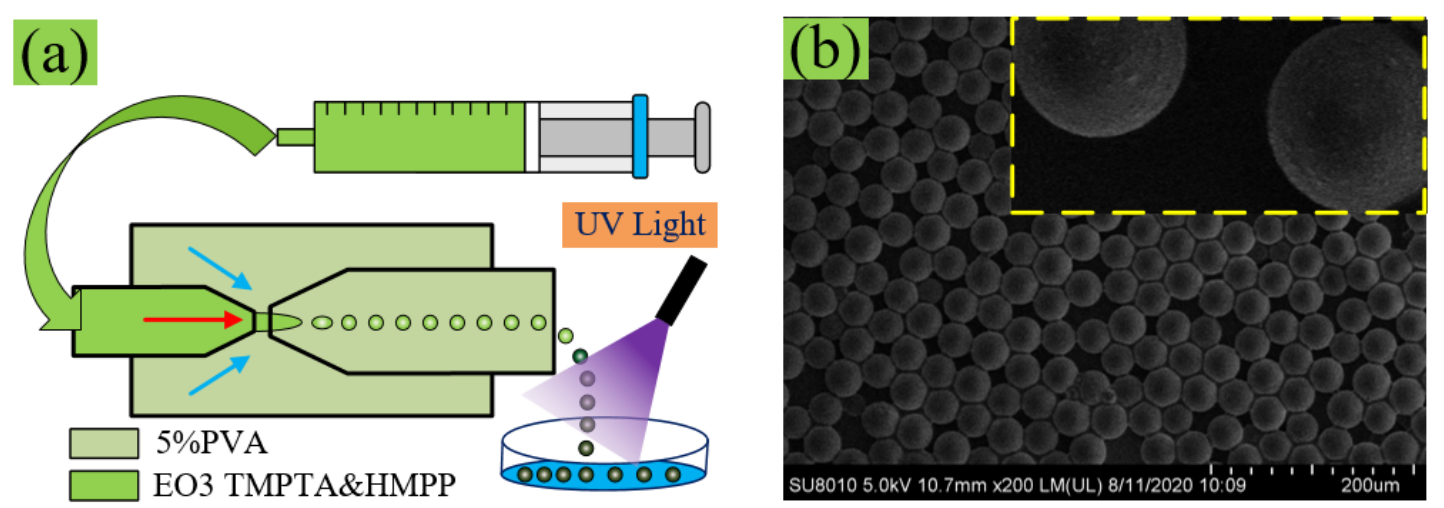

Figure S10 Fabrication of microdroplet. (a) Sketch of setup for droplet generation. (b) SEM image of fabricated droplets.

In the droplet generation, continuous phase consists of $10 \mathrm{wt} \%$ pluronic f108 (Aladdin) aqueous solution and dispersed phase is the Ethoxylated (3) trimethylolpropane triacrylate (EO3 TMPTA, Sigma). ${ }^{5}$ 
Section 13. Formation process of droplet system

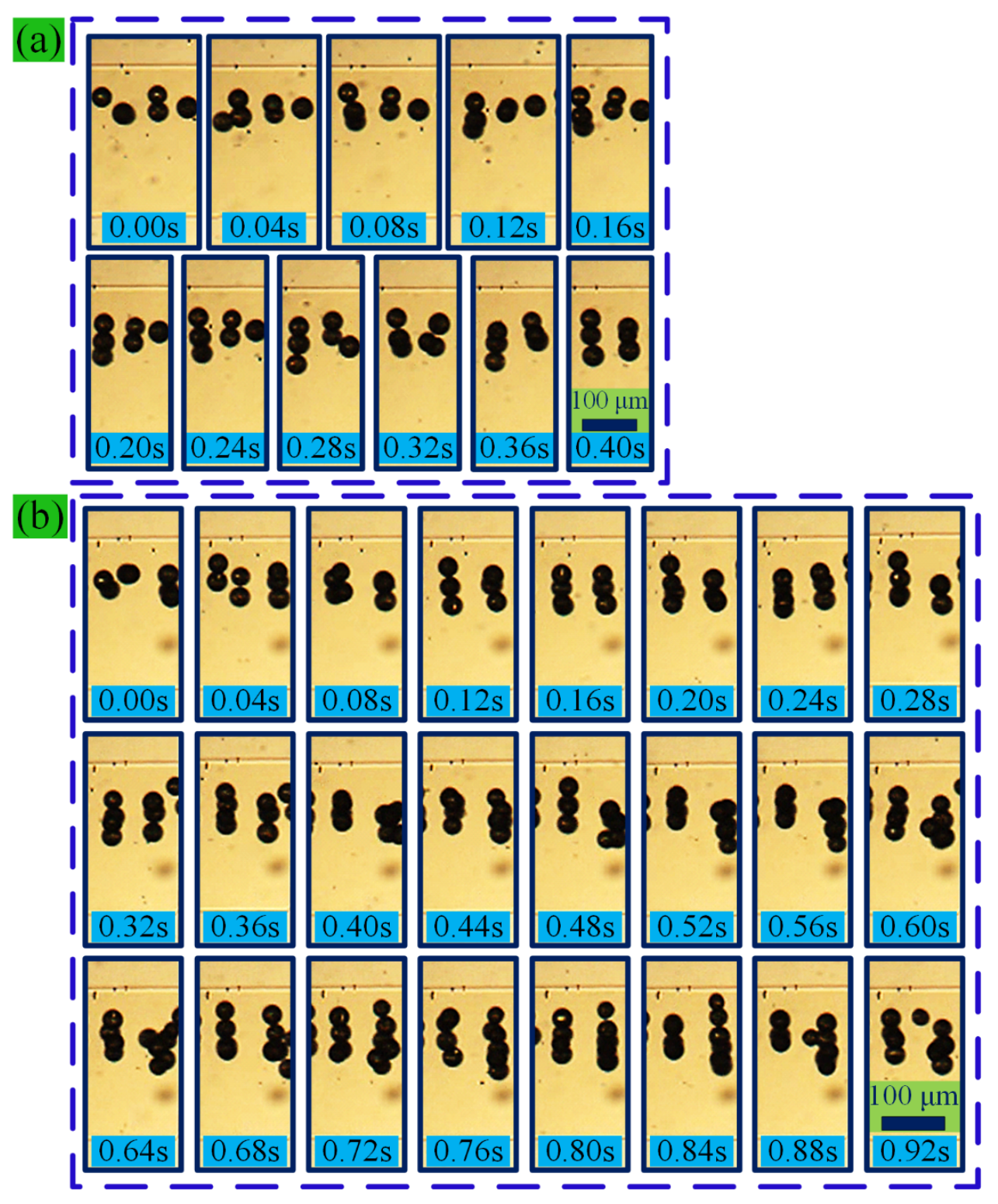

Figure S11 Time-lapse images demonstrating the formation process of droplet system. (a) Formation of three-droplet system. (b) Formation of four-droplet system. 
Section 14. Flow-field distribution in the microchannel

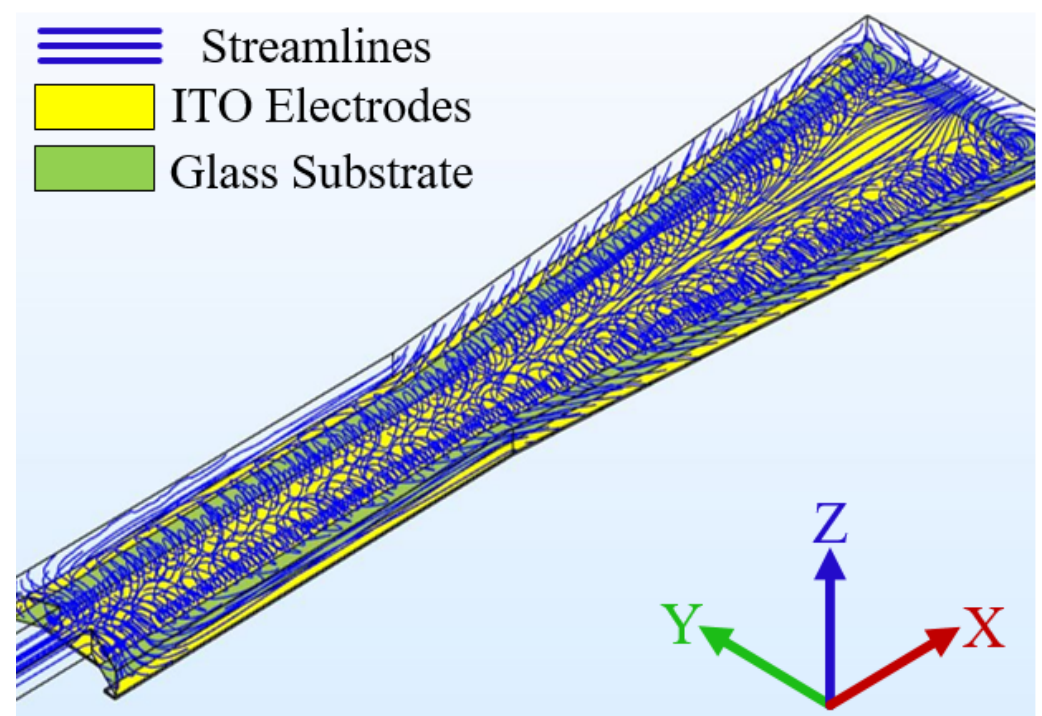

Figure S12 Flow-field distribution in microchannel. 


\section{Section 15. 3-D surface plots}

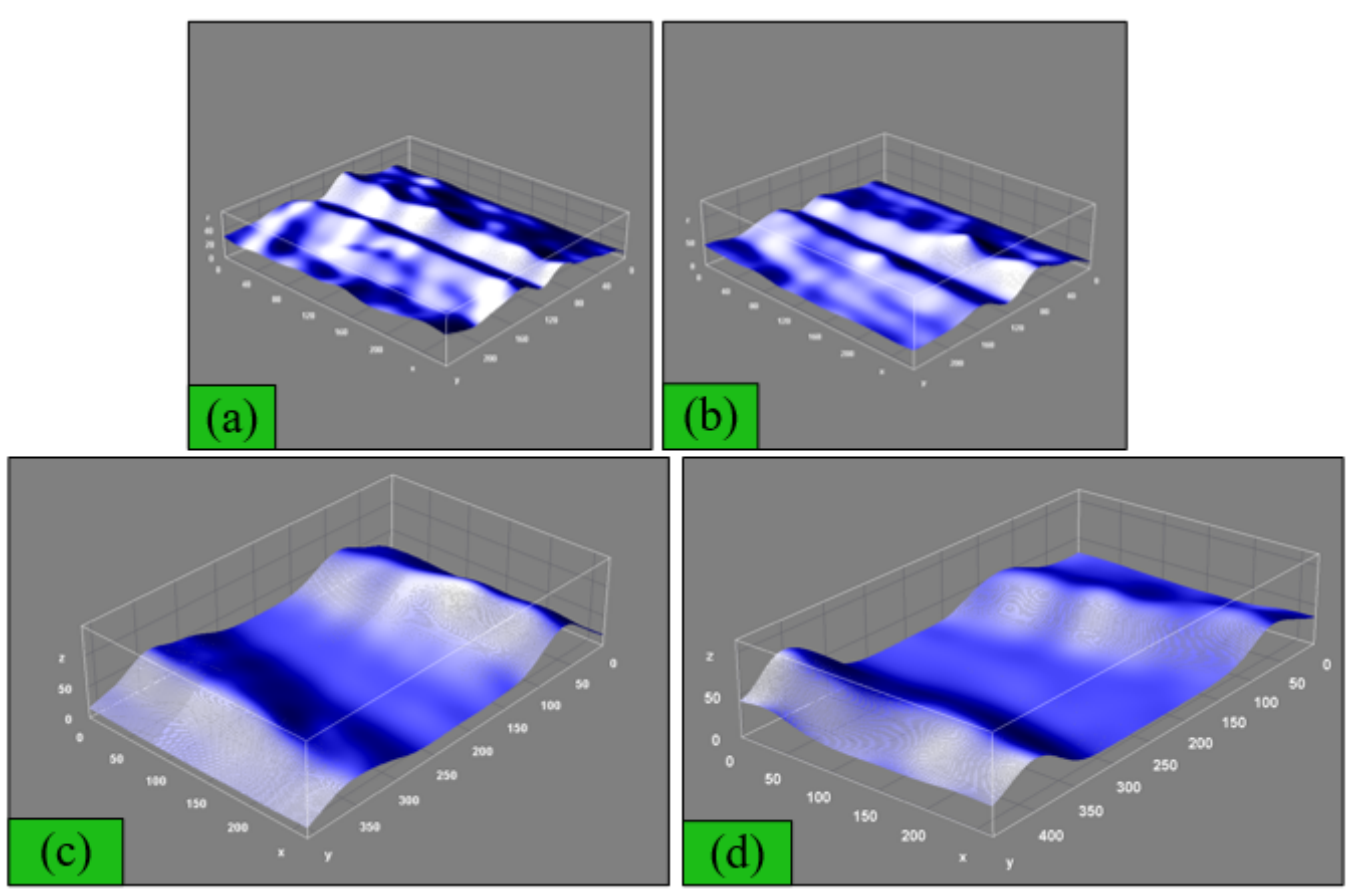

Figure S13 3-D surface plots. (a-d) 3-D surface plot exhibiting separation fluorescent images defined in Figure $4 d(I-I V)$ of manuscript. 
Section 16. Photographs of collected Oocystis sp.

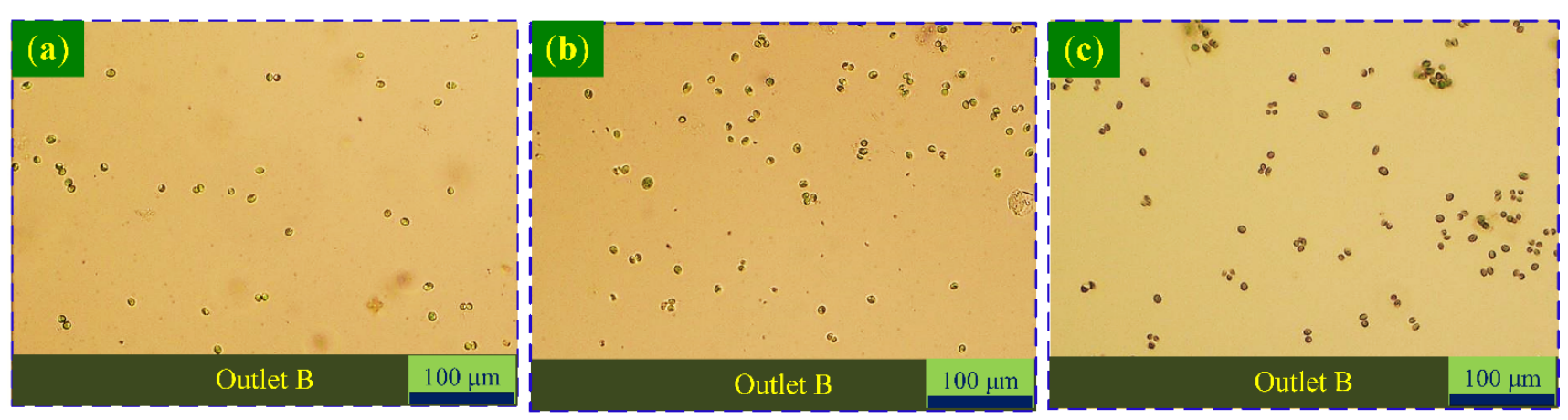

Figure S14 Oocystis sp. collected from outlet B under different conditions. 


\section{Section 17: Supplementary Movies}

Video S1: Collective motion of silica particles in particle-fluid induced-charge electroosmotic vortices.

Video S2: Collective motion and transport of 20- $\mu \mathrm{m}$ Polystyrene particles in particle-fluid inducedcharge electroosmotic vortices.

Video S3: Moving performances of two- and three-droplet system actuated by particle-fluid inducedcharge electroosmotic vortices.

Video S4: Separation of nanoparticles via induced-charge electroosmotic vortices at $A=11 \mathrm{~V}, f=50 \mathrm{~Hz}$, and $u=90 \mu \mathrm{L} / \mathrm{h}$.

Video S5: Isolation of Chlorella vulgaris via induced-charge electroosmotic vortices at $A=6 \mathrm{~V}, f=200$ $\mathrm{Hz}$, and $u=90 \mu \mathrm{L} / \mathrm{h}$.

Video S6: Isolation of Oocystis sp. via induced-charge electroosmotic vortices at $A=6 \mathrm{~V}, f=300 \mathrm{~Hz}$, and $u=90 \mu \mathrm{L} / \mathrm{h}$. 


\section{Reference}

(1) Ren, Y.; Liu, W.; Wang, Z.; Tao, Y. Induced-charge electrokinetics in rotating electric fields: A linear asymptotic analysis. Physics of Fluids 2018, 30, 062006.

(2) Squires, T. M.; Bazant, M. Z. Induced-charge electro-osmosis. Journal of Fluid Mechanics 2004, 509, 217-252.

(3) Ren, Y.; Liu, W.; Jia, Y.; Tao, Y.; Shao, J.; Ding, Y.; Jiang, H. Induced-charge electroosmotic trapping of particles. Lab on a chip 2015, 15, 2181-2191.

(4) Lewpiriyawong, N.; Kandaswamy, K.; Yang, C.; Ivanov, V.; Stocker, R. Microfluidic Characterization and Continuous Separation of Cells and Particles Using Conducting Poly(dimethyl siloxane) Electrode Induced Alternating Current-Dielectrophoresis. Analytical chemistry 2011, 83, 9579-9585.

(5) Zhang, K.; Ren, Y.; Tao, Y.; Deng, X.; Liu, W.; Jiang, T.; Jiang, H. Efficient particle and droplet manipulation utilizing the combined thermal buoyancy convection and temperature-enhanced rotating induced-charge electroosmotic flow. Analytica chimica acta 2020, 1096, 108-119. 